\title{
La différence sexuelle au cabinet des anthropologues parisiens à la fin du XIX siècle
}

Eva Rodriguez

\begin{abstract}
[Résumé] Dans le dernier tiers du XIX siècle, l'anthropologie biologique, et a fortiori, l'anthropométrie, se consacre en France comme une démarche scientifique que la standardisation des méthodes et des instruments doit garantir. Reposant sur un naturalisme direct, elle se présente comme une entreprise d'objectivation et de hiérarchisation des différences, principalement raciales. Dans cet article, il s'agit d'étudier comment, dans ce corpus anthropologique (notamment dans les travaux de la Société d'Anthropologie de Paris) la différence sexuelle, qui pouvait troubler les classifications raciales, va être redéfinie comme une différence variable selon les races, au risque de saper les fondements naturalistes sur lesquels repose cette différence des sexes.

Mots-clés : Anthropométrie, différence sexuelle, race, France, fin de siècle.
\end{abstract}

[Abstract] In the last third of the 19th century in France, Biological Anthropology, and even more so Anthropometry, is confirmed to be a scientific approach that the standardization of methods and instruments must guarantee. Based on a direct naturalism, it claims to be an initiative of objectifi- cation and hierarchization of differences, mainly racial ones. In this paper, I will study how, in this anthropological corpus (especially in the work of the Société d'Anthropologie de Paris), sexual difference, disturbing racial classifications, was re-defined as a variable difference dependent of race, at the risk of undermining the naturalistic foundations underlying this sexual difference.

Keywords : Anthropometry, sexual difference, race, France, fin de siècle.

En 1904, dans une communication prononcée à la Société d'Anthropologie de Paris (SAP) intitulée "Sur l'aspect négroïde de quelques crânes trouvés en France » et publiée dans les Bulletins et Mémoires de la Société d'Anthropologie de Paris (BMSAP), l'anthropologue Léonce Manouvrier reprend, pour les infirmer, les conclusions émises par deux collègues au sujet de quatre crânes préhistoriques qualifiés de « types négroïdes ». Deux de ces crânes, trouvés à Menton, avaient été analysés, entre autres, par René Verneau (Verneau, 1902; Gaudry, 1902); les deux autres, trouvés en Bretagne et conservés dans les collections de la SAP, avaient été étudiés par Georges Hervé (Hervé, 1903). Les deux crânes provenant des côtes armoricaines étaient présentés comme étant deux crânes féminins; les crânes analysés par Verneau appartiendraient à un adolescent masculin et à une vielle femme. Pour ces deux auteurs, ces 
crânes pourraient participer d'une découverte majeure et surprenante : les « races européennes actuelles » seraient reliées à un «type ethnique négroïde » du quaternaire, méconnu jusqu'alors. Ces crânes auraient ainsi la particularité de présenter un ensemble de caractères considérés comme le propre du «type négroïde ». La liste, que nous résumons à grands traits, est la suivante: une dolichocéphalie ${ }^{1}$; un prognathisme ${ }^{2}$ sous-nasal - notamment décrit chez les trois sujets considérés comme féminins, le crâne du sujet adolescent étant en moins bon état - ; une platyrhinie ${ }^{3}$; une faible courbure sous-occipitale ${ }^{4}$ ou encore une forte dentition " que l'on n'hésitera pas à rapprocher de celles d'une des races qu'on s'accorde à placer vers les derniers échelons de l'échelle nigritique, [...] les Australiens, [...] et qui n'a rien à voir avec les européens actuels ${ }^{5}$ ».

Dans sa communication, Manouvrier met en doute les conclusions de ses collègues, en arguant que s'agissant de crânes féminins, on ne peut considérer avec certitude qu'il s'agisse de crânes «négroïdes », malgré leurs apparences, parce qu'ils se caractériseraient précisément par les mêmes traits: dolichocéphalie-non exclusive aux « crânes négroïdes »; aplatissement du vertex ${ }^{6}$, « un des caractères sexuels du crâne les plus constants » (Manouvrier, 1904, 120) ; et surtout, un prognathisme sous-nasal visiblement prononcé. Or, selon Manouvrier, ce prognathisme féminin est en réalité visuellement accentué par la faible proéminence naso-frontale des femmes, elle-même en rapport avec la petite taille et la masse squelettique de bon nombre de sujets féminins.

On sait que les recherches de Topinard firent considérer la femme comme plus prognathe que l'homme, surtout à cause de la proéminence sous-nasale. Ce résultat était en contradiction avec celui que j'obtins plus tard au sujet de l'indice crânio-mendibulaire. Mais je ne tardai pas à démontrer que la supériorité du prognathisme féminin résultait simplement de ce que l'on avait négligé, dans la technique des mensurations, un fait important : c'est qu'une bonne partie du prognathisme masculin se trouve réalisée par la proéminence fronto-nasale, tandis que le prognathisme féminin, n'étant pas dissimulé par cette proéminence, apparaît tout entier là où il se produit presque exclusivement dans la plupart du cas, c'est-à-dire à la région sous-nasale. (Manouvrier, 1904, 121)

\footnotetext{
${ }^{1}$ Désigne un crâne allongé.

${ }^{2}$ Désigne un degré important d'avancement des mâchoires par rapport au crâne.

${ }^{3}$ Désigne un nez large et plat.

${ }^{4}$ Désigne la zone postérieure-inférieure du crâne. Cette faible courbure est censée dénoter un « poids cérébral relatif peu élevé » (Manouvrier, 1904).

${ }^{5}$ Verneau à propos des analyses de leurs dentitions par Albert Gaudry («Discussion » dans Hervé, 1903, 438).

${ }^{6}$ Désigne le sommet de la tête.
} 
Se référant à ses propres études et aux cas présentés par Verneau et Hervé, Manouvrier en conclut que le prognathisme féminin peut donner aux femmes une apparence « négroïde », aujourd'hui encore ajoute-il, alors même qu'il n'en serait rien.

Cet exemple de débat, loin d'être isolé dans la littérature anthropométrique de l'époque, illustre assez bien l'état des discussions au tournant du XX siècle. Dans l'anthropologie fin de siècle, l'anthropométrie - et a fortiori la craniométrie - connaît un développement spectaculaire et devient «la première théorie biologique s'appuyant sur des données quantitatives en grand nombre»(Gould, 1997, 64). L'obsession des mensurations physiques et l'amassement de dizaine de milliers de pièces ostéologiques et anatomiques (Dias, 1989), atteignent leur point culminant. Les méthodes, les techniques et les instruments de mesure deviennent ainsi des enjeux cruciaux dont témoignent les nombreux débats à leurs sujets. Ils doivent permettre d'asseoir l'autorité scientifique de l'anthropologie comme discours de vérité sur «l'Homme».

Ce qui frappe dans l'exemple ci-dessus, c'est que les mêmes traits, par une forme d'analogie, semblent définir pour Verneau et Hervé le «type négroïde », indépendamment du sexe, tandis que pour Manouvrier, ils constituent le propre du sexe féminin, sans précision de «type racial».

Nancy Stepan (1986) a déjà analysé, dans ses travaux portant sur le concept d'analogie dans les sciences, comment dans les discours scientifiques, l'attention portée au XIX ${ }^{\mathrm{e}}$ siècle à la différence sexuelle avait été construite sur un modèle analogue à celui des différences raciales, les femmes des « races les plus civilisées » partageant des traits communs avec les hommes des « races les moins civilisées ». Par ailleurs, à propos des études de squelettes au XVIII ${ }^{\text {e }}$ Londa Schiebinger (1993) a montré que les théories sur les différences raciales avaient été élaborées à partir de l'examen de sujets masculins ; tandis que l'étude de la différence sexuelle s'était concentrée sur des sujets de la classe moyenne européenne. En outre, les différences raciales et sexuelles reposaient sur deux visions contradictoires de la nature : la différence entre les sexes répondait à une différence biologique de type radicale et complémentaire ; la différence entre les races était basée sur une hiérarchie graduelle et linéaire.

Or, ce que la discussion citée en exemple semble montrer précisément, c'est que les procédés d'identification des "types raciaux » sont troublés par les caractères sexués et vice versa. Pour l'étude du genre humain du point de vue de sa diversité raciale, la différence sexuelle constitue un problème qui implique sa redéfinition. C'est ce que nous tenterons d'analyser dans cet article en interrogeant l'élaboration d'un discours sur la différence sexuelle relativement à l'entreprise anthropologique de division de l'humanité en « races». Dans un premier temps, nous étudierons le rôle des méthodes pratiques d'acquisition des connaissances et d'objectivation des corps (observés et observateurs) dans les savoirs anthropologiques de la SAP à la fin du XIX $\mathrm{X}^{\mathrm{e}}$ siècle. Puis, dans un second temps, nous nous intéresserons au cadre théorique évolutionniste dans lequel ces classifications s'enracinent. 
Le corpus étudié est principalement composé des publications des membres de la SAP, entre autres, dans les revues qui privilégient l'étude des caractères physiques de "l'Homme » plutôt que ses expressions morales ou culturelles. Il s'agit principalement des BMSAP, publication fondée en 1859 ; de la Revue d'Anthropologie (RA), fondée en 1872 pour devenir un espace d'expression plus large que les BMSAP; et de L'Anthropologie (fusion en 1890 de la RA, Des Matériaux pour l'histoire naturelle et primitive de l'homme et de La Revue d'Ethnographie). Par ailleurs, notre réflexion est redevable des travaux d'histoire des sciences humaines et des outils des études sociales et féministes des sciences.

\section{Observer et objectiver les corps}

Tout au long de la deuxième moitié du XIX ${ }^{\mathrm{e}}$ siècle en France, l'anthropologie, en tant qu'unité de savoir ordonné qui se donne pour objet «l'étude du groupe humain considéré dans son ensemble, dans ses détails et dans ses rapports avec le reste de la nature » (Broca, 1871, 1), s'institutionnalise comme champ d'investigation scientifique et s'autonomise - relativement - des autres branches des savoirs voisins (ethnologie, archéologie, préhistoire, linguistique, géographie, sociologie, biologie, etc.). On assiste, en marge des universités, à la multiplication de sociétés savantes, revues, structures d'enseignements, laboratoires, musées, etc., et plus largement, au développement d'une anthropologie « au sens large», d'un savoir sur «l'Homme » qui englobe l'étude de ses dimensions à la fois biologiques, physiques, morales et culturelles.

Une des particularités de l'anthropologie française fin de siècle, que nous retiendrons ici, est d'avoir été majoritairement pratiquée par des anthropologues de formation médicale (Dias, 1991, 39-40 ; Vallois, 1959), ayant tenu à faire de «l'anthropologie biologique " proche de l'anatomie comparée, la branche la plus «scientifique » de l'anthropologie. Cette particularité explique certainement le privilège accordé à l'étude des caractères physiques dans l'anthropologie française. Elle permet également, en partie, de comprendre l'attachement aux démarches méthodologiques favorisant l'observation.

$\mathrm{Au}$ sein de la SAP, c'est le laboratoire, que l'on peut définir comme un espace public discipliné, d'accès restreint, où se réalisent des pratiques expérimentales, discursives et sociales, contrôlées collectivement par des membres "compétent·e.s » (Shapin \& Schaffer, 1993, 42) qui va constituer le lieu privilégié de l'étude des corps physiques. Cet espace impose et garantit une discipline d'un genre particulier auquel les scientifiques sont les seuls à avoir accès. L'anthropologie française est alors une science do- 
minée par les anthropologues de cabinet (armchair anthropologists 7). Ils deviennent les dépositaires d'un savoir scientifique légitime, par opposition aux voyageurs, explorateurs, militaires qui se contenteront de recueillir, rapporter ou envoyer des matériaux et des données provenant de «l'ailleurs »-en suivant de nombreuses instructions élaborées adhoc - afin que soit étudiée en laboratoire, de façon approfondie, la diversité du vivant. Comme le rappellent Adolphe Bertillon et Arthur Chervin dans leurs Instructions anthropométriques : «il faut d'abord considérer qu'on ne peut pas demander à un voyageur toutes les mensurations qu'il est possible de recueillir et qu'on recueille tranquillement, à loisir, dans un laboratoire » $(1909,1)$.

Le laboratoire est ainsi un espace clé de l'histoire de l'anthropologie en France, longtemps laissé dans l'ombre (Vallois, 1940). C'est dans le laboratoire de la SAP fondé en 1868 par Paul Broca puis installé en 1876 au réfectoire des Cordeliers à Paris - que l'on apprend la "rigueur » du travail et de la pratique anthropométrique; c'est aussi là que les corps scientifiques s'épuiseront à la tâche. "La science de l'homme » doit tirer ses conclusions d'une étude précise, minutieuse, patiente et laborieuse, réalisée à l'aide d'instruments et de méthodes homogénéisées - étude que les conditions offertes par le laboratoire favorisent.

L'anthropologie se présente donc comme une science d'observation, «l'emploi de méthodes rigoureuses d'observation étant ce qui fait sa force et lui donne un caractère positif " (Broca, 1878, 19). Si la vision - le regard éduqué - apparaît comme le sens le plus important dans la pratique de l'observation scientifique, c'est précisément parce qu'au contraire du toucher, par exemple, elle introduit une distance entre le sujet observant et le sujet observé. Si on peut voir sans être vu, on ne peut toucher sans être touché en retour8. En outre, cette distance sera matérialisée par l'usage des instruments qui vont servir d'intermédiaires entre sujets de connaissance et objets de connaissance. En effet, dans la pratique anthropométrique, on peut dire que l'observation introduit une double objectivation des corps : objectivation du corps observateur qui ne doit rien laisser transparaître de sa subjectivité ; objectivation des corps observés, dont l'inertie du cadavre sera finalement préférée à l'indiscipline du corps vivant.

En effet, face à des sujets pouvant se montrer peu «coopératifs», "insoumis» " craintifs ", « impatients », « indisciplinés », dont «il est arrivé bien des fois qu'[il] refus[e], non seulement de laisser achever l'opération, mais encore, de laisser continuer les autres mensurations, et même qu'il a pris la fuite, entraînant avec lui ses cama-

\footnotetext{
${ }^{7}$ Sur la distinction entre armchair anthropologists et anthropologues de terrains, voir entre autres, Blanckaert (dir.) (1996).

${ }^{8}$ La distinction entre voir et toucher est développée de manière approfondie dans Dias (2004).
} 
rades » (Broca, 1879, 43) ; autrement dit, face à des sujets opposant une résistance9, les corps inertes, morts, s'avèrent bien plus adaptés aux pratiques de la science anthropométrique. Ainsi, dans ses Instructions générales pour les recherches anthropologiques à faire sur le vivant, Broca prévenait: "les voyageurs ne décideront pas toujours aisément les indigènes à se laisser mouler la tête. Il faut d'ailleurs une certaine habileté pour faire cette opération sur le vivant. Mais il n'est personne qui ne puisse mouler la tête d'un cadavre et lorsque l'occasion s'en présente, on ne manquera pas de la saisir » (Broca, 1879[1864], 7).

Concernant l'objectivation du "soi scientifique» (Daston \& Galison, 2012), il faut d'abord rappeler qu'entre les débuts de la SAP (1859) et 1890, une seule femme est membre de la société ; entre 1890 et 1920, seulement six femmes auront l'occasion de présenter leurs travaux ${ }^{10}$. Le corps générique de l'observateur est, implicitement ou explicitement, exclusivement en tous les cas, un corps masculin.

Par ailleurs, ce corps observateur fait usage de ses sens, et ces sens sont faillibles et limités (Dias, 2004). Les instruments techniques, les méthodes standardisées de récolte de données, de mesure, de reproduction (photographies, moulages) ou de conservation vont jouer un rôle fondamental quant à la légitimation de la pratique anthropologique comme science. Ils se présentent comme le moyen de dépasser les limites physiques des corps observateurs en amplifiant la capacité de leurs organes et de leurs sens. Ils contribuent à créer de nouveaux objets auparavant inexistants pour ces sens, à travers le développement d'une nouvelle géométrie des formes corporelles observées qui s'accompagne d'un lexique à forte valeur sémiotique (Blanckaert, 2009, 25) : angles faciaux divers, indices corporels, nouveaux rapports entre points de repères ostéologiques, etc. Ils constituent également une manière de tenir à distance l'objet d'étude, en imposant un éloignement physique entre sujet et objet de la connaissance, médiatisé par l'instrument. L'objectif est de produire un sujet de connaissance désincarné qui supprime l'interprétation et l'intervention subjective afin « d'effacer la personnalité de l'observateur » (Topinard, 1885a, 219) : c'est l'instrument qui parle. Enfin, pour que l'observation soit validée, il faut qu'elle puisse être partagée ; il faut la « neutraliser ». C'est cette neutralisation que les instruments doivent réaliser en assurant la standardisation, l'homogénéisation des méthodes, notamment à travers la modélisation ou la numérisation du langage scientifique. De façon générale, «l'homme » des sciences anthropométriques incarne à merveille ce savant décrit par Haraway dont «la distance sans passion est sa plus grande vertu » (Haraway, 2012, 197).

\footnotetext{
${ }^{9}$ Voir à ce sujet, par exemple, le sous-chapitre " un obstacle épistémologique : l'enquêté », dans Blanckaert (2009, 195-200).

${ }^{10}$ Au sujet des femmes dans les sociétés savantes au XIX ${ }^{\mathrm{e}}$ siècle, voir Charron (2013).
} 
Les Instructions (1879 [1864]) de Broca, ont ainsi pour vocation d'indiquer les instruments aptes aux conditions du terrain et du voyage, les procédés de mensuration invariables, des échelles chromatiques numérisées pour la couleur des yeux, cheveux, peaux, etc. De multiples instruments (craniographes, stéréographes, endographes, pachymètres, goniomètres, céphalomètres, etc.) vont être fabriqués, débattus ; ils doivent contribuer à produire les données les plus fiables et irréfutables possibles. Ils vont donner lieu à de nombreuses controverses et discussions - à l'image de celle que nous citions en introduction entre Topinard et Manouvrier à propos de la façon de calculer le degré de prognathisme des hommes et des femmes.

Ce que cet attachement à la discussion et au débat permet de comprendre, c'est qu'au sein de la SAP, la démonstration, la méthodologie et le niveau de preuves importent plus que les théories produites ou les dogmes qui les soutiennent. La hiérarchisation inégalitaire des « races » ou des « sexes » constitue un acquis profond et un accord a minima, peu (ou pas) remis en question. Les anthropologues s'attardent moins sur les implications politiques des hiérarchies qu'ils produisent que sur les méthodes avec lesquelles ils les produisent. Ainsi, les débats portent plus sur les méthodes de classification et de typologisation que sur les conclusions auxquelles elles mènent, c'est-à-dire la hiérarchisation des êtres. C'est pourtant à l'intérieur de ces discussions que ces hiérarchies s'enracinent. Et c'est au détour des discussions portant sur les méthodes et les pratiques de typologisation raciale que l'on peut retracer aussi, en filigrane, comment se construit un discours sur la différence sexuelle, alors même qu'elle apparaît souvent comme un objet secondaire dans la littérature anthropométrique.

\section{Corps de femmes, corps d'hommes : quelles différences?}

Depuis le XVIII ${ }^{\mathrm{e}}$ siècle, pour les médecins, naturalistes et anthropologues, la différence sexuelle s'exprime dans toutes les parties du corps et se lit surtout dans ceux des femmes.

«La femme n'est pas femme seulement par un endroit», dit Roussel, «mais par toutes les faces par lesquelles elle peut être envisagée ». Et Virey, quarante ans plus tard: "Les différences sexuelles ne sont point bornées aux seuls organes de la génération, chez l'homme et chez la femme, mais toutes les parties de leur corps, celles même qui paraissent indifférentes aux sexes en éprouvent quelque influence. " C'est la grande découverte du temps et des anatomistes, en foi de quoi on cherche à établir les spécificités du corps féminin - et on insiste sur deux traits : la faiblesse et la prédestination à la maternité. (Knibiehler, 1976, 829)

On retrouve tout au long du XIX ${ }^{\mathrm{e}}$ siècle cette idée, reprise maintes fois dans les traités médicaux, que «la » femme - surtout - est en toute part femme: un poumon, un 
rein, une jambe, un bassin, un cerveau, un fémur, un nez, un pied, une mâchoire etc. ; toute partie du corps est sexuée.

Dans une communication présentée par Topinard à la SAP, en 1885 (Topinard, 1885b), portant sur les procédés de mensuration des os longs (fémur, tibia, humérus, etc.) pour restituer la taille des races préhistoriques, celui-ci s'explique longuement à propos des méthodes utilisées. Dans cet article assez technique, on peut lire, le passage suivant :

Pour commencer, il faut s'attacher à séparer les sexes, autrement dit [...] établir trois catégories lorsque l'ensemble des os, le crâne et le bassin surtout, ou quelques particularités de la sépulture, ne leur apprendront pas le sexe avec certitude, à savoir: une première d'os longs du sexe masculin, absolument certains à leurs yeux; une seconde d'os féminins, absolument certains, et une troisième d'os incertains. Si la détermination du sexe est quelquefois difficile sur le crâne, elle l'est davantage sur les os, et cette phase préalable de la mensuration est la seule délicate. (Topinard, 1885b, 73)

S’en suit une discussion longue, crispée, avec Clémence Royer et Manouvrier entre autres, au sujet, toujours, des méthodes. Clémence Royer rétorque par exemple à Topinard qu' « en éliminant les cas douteux au point de vue du sexe, on accentue les caractères sexuels, car les cas restants sont les plus masculins et les plus féminins. Il s'en suivrait qu'on élèverait la moyenne de la taille des hommes et qu'on diminuerait celle des femmes » (« Discussion » dans Topinard, 1885, 78). Cependant, les conséquences de ce tri sélectif opéré dans le classement des os en fonction du sexe, une forme de biais androcentrique, n'est ni l'enjeu, ni l'objet de la discussion.

Le discours anthropologique majoritaire s'accorde pour dire que les femmes ont globalement des cerveaux, des crânes ou des os plus petits et plus légers que les hommes, en relation avec leur différence et leur infériorité. Et si ces mesures peuvent être questionnées en fonction des procédés de mensuration, l'infériorité postulée des femmes n'est pas pour autant remise en cause. Par exemple, dans ses travaux, Manouvrier tente de prouver que les deux sexes s'équivalent quant au développement cérébral (1902, 1909). Cependant, pour lui, la différence sexuelle ne disparaît pas pour autant, elle s'exprime ailleurs : «la femme » présente sous le rapport de la nutrition une supériorité en lien avec ses fonctions maternelles, tandis que «l'homme» l'emporte sous le rapport du développement et de l'énergie musculaire (1902). En outre, explique-t-il, « les femmes sont des êtres plus grêles, dont les tissus musculaires et osseux sont moins développés »; ou encore - et il est important de souligner ici l'usage récurrent de l'idée de "développement »: il considère que les femmes ont "un plus grand développement des viscères abdominaux, comparativement au développement des systèmes osseux et musculaire »(Demonet, 1905). Il sera d'ailleurs un grand défenseur de l'argument d'une complémentarité des sexes contre l'idée d'égalité (1909).

La deuxième moitié du XIX ${ }^{e}$ siècle est ainsi caractérisée par l'attrait des chiffres, par la croyance que la mesure rigoureuse, la normalisation des méthodes et la statistique doivent garantir la justesse des faits observés et décrits (Gould, 1997, 106). Mais aux 
côtés de cette "quantification », l'évolutionnisme "spéculatif », influencé par les récentes théories sur le développement embryologique, est le paradigme triomphant. En embryologie, une nouvelle thèse - la thèse épigénétique - se substitue à la thèse de la préformation qui considérait le germe initial comme une forme miniature - tous les organes seraient présents à l'origine - de la forme finale. La nouvelle thèse épigénétique implique, au contraire, de penser qu'il y a des transformations successives et qualitatives dans le développement organique entre le germe initial et la forme finale ${ }^{11}$. En généralisant cette loi épigénétique, un certain évolutionnisme spéculatif établit que le développement d'un organisme individuel et d'un organisme social se réalise par un mécanisme identique qui consiste dans le passage d'une structure homogène à une structure hétérogène, d'une structure simple à une structure complexe. La différence sexuelle, retravaillée à l'aune de ces nouvelles théories est nouvellement appréhendée comme une interruption prématurée du développement ; comme résultant «de ce que l'arrêt de l'évolution individuelle est un peu plus précoce chez la femme que chez l'homme » (Spencer, 1903) ${ }^{12}$. C'est dans ce cadre de pensée évolutionniste que la différence sexuelle va principalement être analysée.

Dans l'anthropologie, en tant que «branche de l'histoire naturelle qui traite de l'homme et des races humaines » (Topinard, 1895, 2), c'est le corps masculin comme corps générique qui est principalement étudié : c'est lui qui incarne les « types ».

Tout d'abord, on n'étudie pas un spécimen femme dans le but de caractériser un quelconque type racial. L'exemple cité en introduction tend ainsi à montrer qu'à vouloir caractériser un "type » à partir de spécimens féminins, on risque surtout de commettre des erreurs. On étudie donc les femmes, dans leurs «spécificités 》 de femmes (menstruations, fertilité, etc.), ou par rapport aux spécimens masculins. La science de l'« Homme » est androcentrique; au fond, «l'Homme» c'est l'homme, elle s'intéresse en tout premier lieu au corps masculin comme corps générique ${ }^{13}$. Les corps féminins sont plus souvent étudiés comme compléments de séries masculines. Ainsi,

\footnotetext{
${ }^{11}$ C'est en partie la tératologie - science des monstres ou des malformations - qui a fourni à l'embryologie ses principales expérimentations pour étayer la thèse épigénétique de formation des organismes. En cherchant à expliquer comment les malformations surviennent, elle a établi que les anomalies résultent d'une interruption dans le développement embryonnaire, et par conséquent, ne préexistent pas à leur formation. Le "monstre humain » était ainsi considéré comme un être altéré, anormal, arrêté ou retardé dans son développement et non plus comme le résultat d'un mode de création absolument différent. Voir par exemple, Canguilhem et al. (1962).

${ }^{12}$ Herbert Spencer (1820-1903) est un des représentants les plus importants de ce courant.

${ }^{13}$ Sur ce point, le titre d'un mémoire de Clémence Royer qui sera refusé, "La femme: l'animal de la création que l'homme connaît le moins », est tout à fait éloquent. Royer fut la première femme membre de la SAP. Elle fut également la première traductrice française et préfacière de L'origine des espèces de Charles Darwin en 1862 [1859].
} 
lorsqu'il s'agit de caractériser des races, s'il existe des études sur des séries de corps, d'organes ou d'os féminins, celles-ci peuvent tout à fait être moins fournies :

La série nécessaire sans laquelle on ne pourrait pas même commencer l'étude d'une race, est celle des sujets mâles et adultes. [...] À quel chiffre doit-on porter le nombre des individus d'une série? La méthode des moyennes est d'autant plus sûre que les faits sont plus nombreux. [...] Une expérience basée sur les faits [...] anthropométriques permet de considérer comme suffisante une série de vingt observations homogènes. [...] La série des femmes adultes est la seconde en importance. Elle est destinée moins à caractériser la race qu'à faire connaître les différences sexuelles qui existent dans cette race, différences dont l'étendue est bien loin d'être la même dans toutes les races. La série des femmes n'est donc qu'un complément des recherches faites sur les hommes: dès lors, elle pourra, si le temps fait défaut être réduite à quinze ou même à dix observations. (Broca, 1879, 186-190)

En outre, alors que la différence sexuelle se présente comme une différence universelle traversant l'humanité, les anthropologues de la SAP n'auront de cesse de dire que cette différence varie et évolue en fonction des " races » et du temps, troublant alors à leur tour ce qui apparaissait comme une dichotomie établie au sein de l'espèce humaine. Loin de revêtir le caractère d'évidence que l'on lui prête, la différence sexuelle, ce qu'il en est de ses définitions, fonctions, et caractéristiques, apparaît bien plus comme un problème que comme une question close, ainsi que le montre la citation suivante de Gustave Le Bon à propos de la taille des crânes et des cerveaux féminins :

Dans toutes les races humaines, le crâne de la femme est moins volumineux que celui de l'homme, mais le degré d'infériorité varie considérablement d'une race à l'autre. L'étude des graphiques que nous avons construits et des chiffres ayant servi à les obtenir nous a conduit à reconnaître ce fait très curieux, que les différences qui existent entre les crânes masculins et féminins d'une même race s'accroissent constamment à mesure qu'on s'élève des races inférieures aux races supérieures, en sorte que, au point de vue de la masse du cerveau, la femme tend à se différencier de plus en plus de l'homme. [...] On arrive à ce résultat curieux que les races où les crânes masculins occupent le haut de l'échelle sont précisément souvent celles où les crânes féminins en occupent les derniers échelons. (Le Bon, 1879, 56-58)

Ainsi, on comprend comment dans les travaux d'anthropologie biologique de la fin du XIX ${ }^{\mathrm{e}}$ siècle, la hiérarchisation des races ne peut être pensée que du point de vue du développement organique et intellectuel des types masculins ; les types féminins venant inverser ou brouiller l'ordre des classifications. L'idée est, d'une part, que la civilisation et le développement des sociétés - caractérisés par le progrès technique induisant une division technique et sociale du travail accrue par l'industrialisation - 
agissent sur l'organisation sociale (la répartition des rôles, des tâches et des fonctions ${ }^{14}$ ), mais aussi sur l'organisation biologique des corps qu'elles forment et déforment. D'autre part, le processus de civilisation social et biologique agit différemment sur les hommes et sur les femmes, à l'avantage des premiers, au détriment des secondes. En effet, en suivant la définition du progrès comme opération diversifiante, passage du simple au complexe ou de l'homogène à l'hétérogène empruntée à l'embryologie du développement, on obtient l'équation suivante : plus une société est organisée de manière complexe dans sa division interne des structures sociales, plus elle est considérée comme développée, et plus les différences (sociales et biologiques) en son sein sont marquées. Les hommes sont donc censés évoluer plus et plus vite que les femmes, ainsi que le corrobore Broca:

Nous trouvons, dans l'état social de la femme civilisée, des conditions de nature à exagérer la différence qui existe naturellement entre le volume de son cerveau et celui du cerveau de l'homme [...] C'est d'ailleurs un fait bien connu que, chez beaucoup de peuples sauvages ou seulement barbares, la constitution de la femme diffère bien moins que chez nous de celle de I'homme [...] On comprendra maintenant pourquoi les différences sexuelles de la capacité crânienne sont beaucoup plus faibles chez les troglodytes de l'homme mort que chez les parisiens modernes. Parmi les autres races inscrites sur le tableau de la page 33, il n'en est qu'une qui puisse être qualifiée de sauvage, c'est celle des Néo-Calédoniens; chez eux la différence sexuelle n'est que de $129 \mathrm{cc}, 52$ [...]. On remarquera que le crâne de ces dernières est à peine plus petit que celui des parisiennes. (Broca, 1873, 46)

Dans cette perspective, les hommes et les femmes doivent donc tendre vers une différenciation prononcée - les femmes pouvant même aller jusqu'à régresser - au fur et à mesure du « développement » des sociétés. La différence sexuelle est donc présentée comme variable. Au regard des différents écrits anthropologiques qui la traitent, elle apparaît, en fin de compte, davantage multiforme dans ses manifestations physiques, qu'uniformément partagée.

\section{Conclusion}

Tout comme la dissection anatomique du cadavre humain a été, depuis la fin du XIII ${ }^{\mathrm{e}}$ siècle, le moyen privilégié de connaissance du corps (Mandressi, 2003; Park, 2009), l'anthropologie biologique propose des critères d'identification à même de regrouper et classer l'humanité en différents types. Les corps organisés ou morcelés semblent alors s'offrir aux regards et aux instruments des anthropologues comme

\footnotetext{
${ }^{14}$ Voir par exemple comment Durkheim reprend à son compte cette idée qu'il emprunte aux anthropologues, notamment à Gustave Le Bon (Durkheim, 1986, 24)
} 
contenant des indices à même de dévoiler la nature humaine, témoignant d'un naturalisme direct (Guillo, 2012) qui établit un continuum entre des caractères biologiques et des hiérarchies raciales principalement, mais aussi sociales et sexuelles. Dans le contexte de l'anthropologie française fin de siècle, l'obsession "quantitative " de l'anthropométrie se présente comme une entreprise d'objectivation des différences qui doit permettre la division de l'humanité en races et leur hiérarchisation sur un axe primitif/civilisé. Dans ce corpus, alors que l'étude de la différence sexuelle n'apparaît pas au premier abord comme un objet concentrant l'attention des anthropologues, voire comme une différence à même de perturber les classifications raciales qu'ils cherchent à établir, elle va être repensée dans le cadre évolutionniste afin de s'intégrer dans les modèles classificatoires. Or, cette nouvelle conception de la « différence sexuelle », redéfinie par les anthropologues et mise au pluriel en parlant de différences sexuelles variables - pour montrer qu'elle est loin d'être la même en fonction des « races »-, propose, « sans le vouloir », un argument en faveur de l'idée que la différence sexuelle est précisément une construction sociale et non pas une dichotomie fondée en nature. Ainsi, il est possible que malgré elle, l'anthropologie biologique, contribue à déconstruire le caractère permanent, naturel et universel de quelque chose qu'on appellerait la différence sexuelle, du point de vue social, mais aussi, et surtout, du point de vue biologique.

\section{Bibliographie}

\section{Sources primaires}

Bertillon A., Chervin A. (1909), Anthropologie métrique : conseils pratiques aux missionnaires scientifiques sur la manière de mesurer, de photographier et de décrire des sujets vivants et des pièces anatomiques [...], Paris : Imprimerie nationale.

Broca P. (1871), Mémoires d'anthropologie, Paris : Reinwald.

Broca P. (1873), « Sur les crânes de la caverne de l’homme mort (Lozère) », RA, t. 2.

Broca P. (1878), «Séance d'ouverture, le vendredi 16 août 1878 », Exposition Universelle, "Congrès international des sciences anthropologiques tenu à Paris du 16 au 21 août $1878 »$ CNAM 8 Xae 253.

Broca P. (1879), Instructions générales pour les recherches anthropologiques à faire sur le vivant, Paris : G. Masson (2e éd.).

Demonet E. (1905), « Recherches sur la capacité vitale absolue et relative suivant le sexe et suivant certaines dimensions du corps », BMSAP, 5e s., t. 6.

Durkheim É. (1893), De la division du travail social, Paris, Felix Alcan. 
Hervé G. (1903), «Crânes néolithiques armoricains du type négroïde », BMSAP, 5e s., t. 4 .

Le Bon G. (1879), "Recherches anatomiques et mathématiques sur les lois des variations du volume du cerveau et sur leurs relations avec l'intelligence », RA, 2e s., t. 2.

Manouvrier L. (1902), « Rapports anthropométriques et sur les principales proportions du corps », BMSAP, 3e s., t 2.

Manouvrier L. (1904), «Sur l'aspect négroïdes de quelques crânes trouvés en France », BMSAP, 5 e s., t. 5.

Manouvrier L. (1909), «Conclusions générales sur l'anthropologie des deux sexes et applications sociales », Revue de l'École d'anthropologie, t. 19.

Royer C. (1874), «L'animal de la création que l'homme connaît le moins » reproduit dans Ducros Albert, Blanckaert Claude, 1991, "'L'animal de la création que l'homme connaît le moins' : Le mémoire refusé de Clémence Royer sur la femme et la natalité ", BMSAP, nouvelle s., t. 3, nº1-2.

Spencer H. (1903), Introduction à la science sociale. Paris : Felix Alcan.

Topinard P. (1885a), Éléments d'anthropologie générale, Paris : Delahaye et Lecrosnier.

Topinard P. (1885b), "Procédé de mensuration des os longs, dans le but de reconstituer la taille », BMSAP, 3e s., t. 8.

Topinard P. (1895), L’Anthropologie, Paris : Reinwald.

Vallois H. V. (1940), « Le Laboratoire Broca », BMSAP, 9e s., t. 1.

Vallois H. V. (1959), « Rapport de M. le professeur H. V. Vallois ».

Verneau R. (1902), « Les fouilles du prince de Monaco au Baoussé-Roussé. Un nouveau type humain », L’Anthropologie, t. 13.

\section{Sources secondaires}

Blanckaert C. (1992), "La science de la femme: une affaire d'hommes » in Collin F. (dir), Le sexe des sciences. Les femmes en plus, Paris : Autrement, pp. 54-62.

Blanckaert C. (dir.) (1996), Le terrain des sciences humaines. Instructions et enquêtes (XVIII ${ }^{e}$-XIX ${ }^{e}$ siècle), Paris : L'Harmattan.

Blanckaert C. (2009), De la race à l'évolution: Paul Broca et l'anthropologie française. Paris : L'Harmattan.

Canguilhem G., Lapassade G., Piquemal J., Ulmann J. (1962), Du développement à l'évolution au XIX $X^{e}$ siècle, Paris : PUF. 
Charron H. (2013), Les formes de l'illégitimité intellectuelle. Les femmes dans les sciences sociales françaises 1890-1940, Paris : CNRS Éditions.

Daston L., Peter G. (2012), Objectivité, Dijon : Les Presses du réel.

Dias N. (1989), "Séries de crânes et armée de squelettes: les collections anthropologiques en France dans la seconde moitié du XIX ${ }^{\mathrm{e}}$ siècle », BMSAP, n. s., t. 1, f. 3-4.

Dias N. (1991), Le musée d'ethnographie du Trocadéro (1878-1908). Anthropologie et muséographie en France, Paris : Éditions du CNRS.

Dias N. (2004), La mesure des sens: Les anthropologues et le corps humain au XIX ${ }^{e}$ siècle. Paris : Éditions Aubier.

Gould S.J. (1997), La Mal-mesure de l'homme. Paris : Odile Jacob.

Guillo D. (2012), « Les usages de la biologie en sciences sociales. Comparaison entre le naturalisme socio-anthropologique du dix-neuvième siècle et celui d'aujourd'hui », Revue Européenne des sciences sociales, 50-1 (en ligne).

Haraway D. (2012[1992]), « Les promesses des monstres. Politiques régénératives pour d'autres impropres/inapproprié-e-s » in Dorlin E., Rodriguez E. (dir.), Penser avec Donna Haraway, Paris : PUF, pp. 159-229.

Knibiehler Y. (1976), «Les médecins et la 'nature féminine' au temps du Code civil», Annales. Économies, Sociétés, Civilisations, 31e année, n4, pp. 824-845.

Mandressi R. (2003), Regard de l'anatomiste: Dissections et invention du corps en Occident, Paris : Seuil.

Park K. (2009), Secrets de femmes: Le genre, la génération et les origines de la dissection humaine, Dijon : Les Presses du réel.

Shapin S., Schaffer S. (1993), Léviathan et la pompe à air. Hobbes et Boyle entre science et politique. Paris : La Découverte.

Schiebinger L. (2004[1993]), Nature's body : Gender in the Making of Modern Science, New Brunswick : Rutgers University Press.

Stepan N. L. (1986), « Race and Gender: The Role of Analogy in Science », Isis, Vol. 77, $\mathrm{n}^{\circ} 2$, pp. 261-277. 